\title{
Transmission Power Line Fault Detection using Convolutional Neural Networks
}

\author{
Kalanidhi $\mathrm{K}^{1}$, Baskar D ${ }^{2}$, Vinod Kumar $\mathrm{D}^{3}$ \\ kalanidhik@gmail.com, basindia.kd@gmail.com,vino.kd@gmail.com \\ ${ }^{1}$ Research Scholar - Electrical \& Electronics Engineering, ${ }^{3}$ Professor \& Head - Biomedical \\ Engineering,Vinayaka Mission's Kirupananda Variyar Engineering College, VinayakaMission'sResearch \\ Foundation (Deemed to be University), Salem, Tamil Nadu, India. \\ ${ }^{2}$ Assistant Professor - Electrical \& Electronics Engineering, Annai Teresa College of Engineering, \\ Viluppuram, Tamil Nadu, India.
}

\begin{abstract}
In an electrical power system, most of the faults occurs in overhead transmission lines because of most of the conductor exposure to the atmosphere. Therefore, Insulated Overhead Conductors (IOCs) are widely used. To overcome this, a robust real-time PD fault analysis system is required. To analyze and classify the raw voltage signal for detection of PD's in IOC's a Convolutional Neural Network (CNN) based fault classification algorithm is proposed in this paper. The CNN is implemented using popular pre-trained CNN architectures such as AlexNet, VGG16 \& ResNet are applied to the voltage signals in the dataset. From the values of Precision, Recall \& F1Score it is observed that ResNet architecture provides the best prediction and classification results.
\end{abstract}

Keywords:Transmission Lines, Fault Analysis, CNN, AlexNet, VGG16, ResNet.

\section{Introduction:}

Partial discharge (PD) can occur in transmission lines. PD represents a breakdown between two conducting electrodes. If PD is not detected, the damage to the transmission line can be disastrous and cause serious safety events. Partial discharge can easily be detected by ultrasound and by a routine preventive maintenance inspection of transmission lines. Over the years, several algorithms have been expanded to automate the classification of Partial Discharge fault sources. There are models that include feature engineering into one framework. They can extract features and automatically create informative depictions of time series and these models are Convolutional Neural Networks (CNNs) and Recurrent Neural Networks (RNN). CNN's based models for the classification of time series has numerous significant benefits over other methods.

\section{VSB - ENET Dataset}

The VSB ENET Centrein the Czech Republicmade several attempts over the past few years to obtain PD signals. They developed a measuring tool that can be placed on IOC's outer cover to measure PDs [1,2]. The configuration can be seen in Fig 1. A single layer coil is wrapped around the IOC to absorb the drift electric field voltage around the IOC, aiming to detect the hazardous PD actions. The voltage signal is further captured by using a capacitancevoltage divider whose output capacity is combined in parallel with the voltage output terminals and an inductor. VSB's approach is economicalthan another alternative solution that uses the Rogowski sensor [3,4,5] to calculate the current in the conductor explicitly.In 2018, VSB issued a dataset for this purpose, including a considerable number of waveform measurements acquired utilizing the system outlined above. The dataset was released by Kaggle[6,7]. 

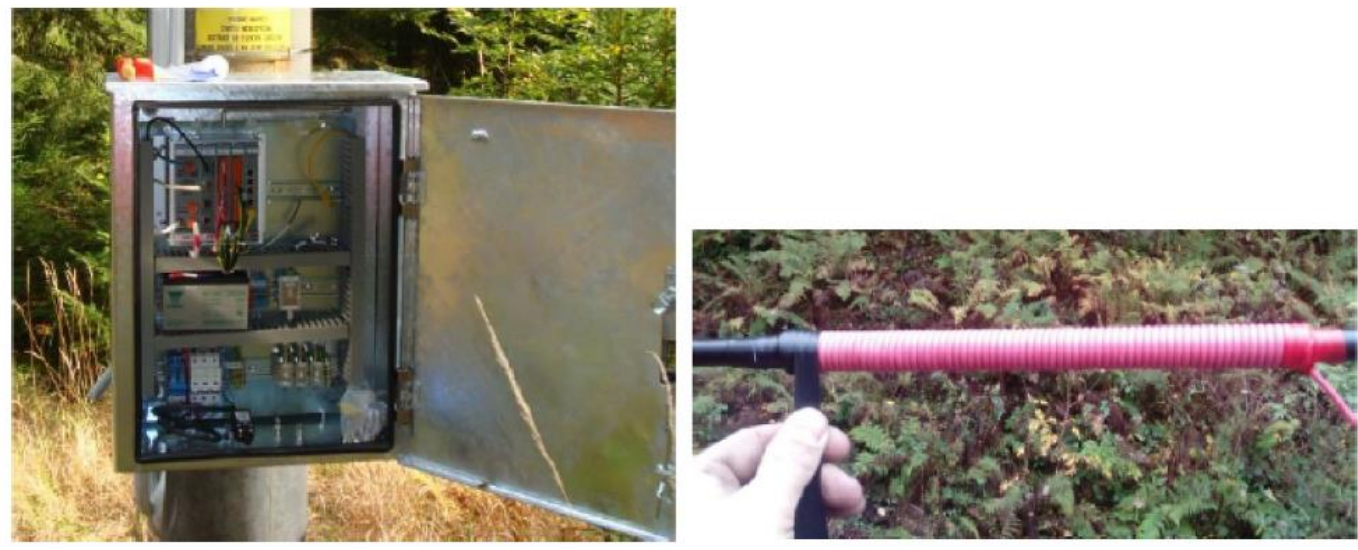

Fig. 1.VSBENET - PD Measuring Device.

The dataset contains 8,711 pre-labelled voltage signals consisting of 8186 Non-PD and $525 \mathrm{PD}$ recorded from more than 20 real-time different locations[8]. Fig 2 presents the waveform of the PD and Non-PD signals. The Non-PD signal has a maximum value of $40 \mathrm{mV}$ and a minimum value of $40 \mathrm{mV}$. The PD signal has a maximum of $60 \mathrm{mV}$ and the minimum value is $-80 \mathrm{mV}$ approximately the fluctuation of the signal rises significantly.
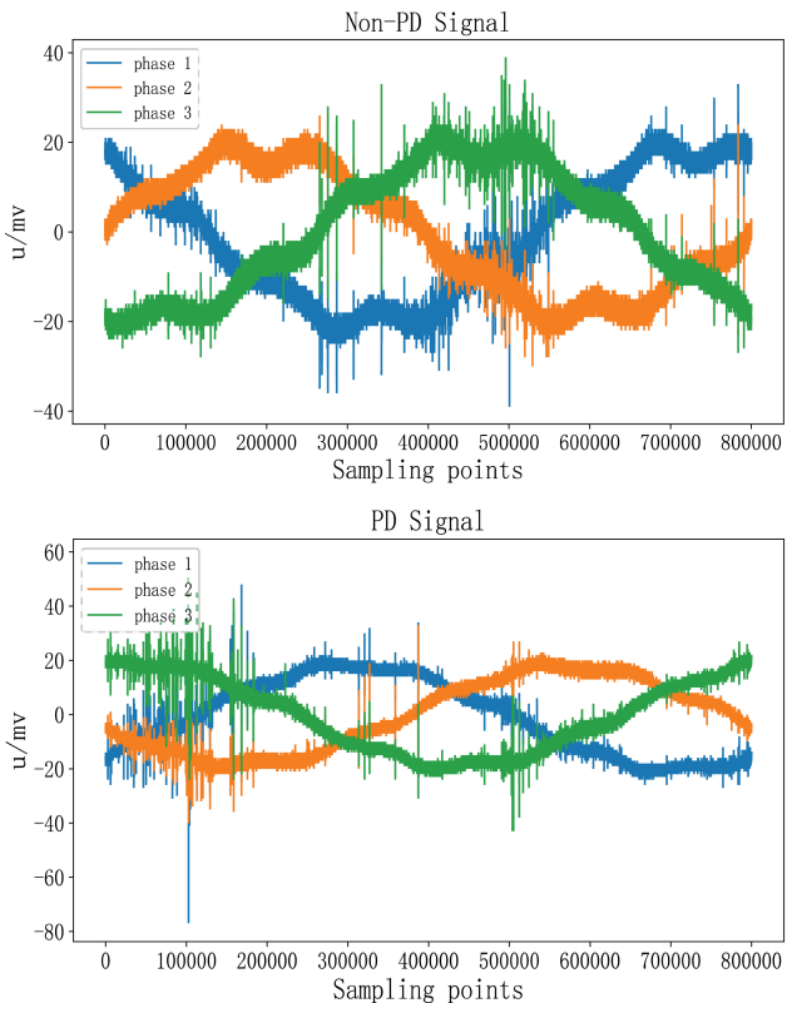

Fig. 2.Waveformof PD and Non-PD Signal records from ENET Dataset.

\section{Performance Metrics}

Standard classification evaluation tools such as Precision, Recall, F1-Score are used to evaluate the performance. True Positive (TP) is the cases in which we predicted YES. The actual output was also YES., False Positive (FP) are the cases in which we predicted YES and 
the actual output was NO., True Negative (TN) is the cases in which we predicted NO. The actual output was NO., and False Negative (FN) is the cases in which we predicted NO. The actual output was YES.

Precision is the number of correct positive results divided by the number of positive results predicted by the classifier. Recall is the number of correct positive results divided by the number of all relevant samples (all samples that should have been identified as positive). F1 score is a measure of a test's accuracy and is defined as the weighted harmonic mean of precision and recall. The range for F1 Score is $(0,1)$.

The precision and recall for PD signals are:

$$
\text { Precision }=\frac{T P}{T P+F P} \quad \text { Recall }=\frac{T P}{T P+F N}
$$

The precision and recall for Non PD signals are:

$$
\text { Precision }=\frac{T N}{T N+F N} \quad \text { Recall }=\frac{T N}{T N+F P}
$$

The F1-Score is the harmonic mean of Precision and Recall:

$$
F 1=2 \frac{\text { Presision } \cdot \text { Recall }}{\text { Presision }+ \text { Recall }}
$$

\section{Convolutional Neural Networks(CNNs)}

Convolutional Neural Networks given in Fig 3. are also know as CNN or ConvNet which are complex feed-forward neural networks. Because of its high accuracy, CNN is used in various applications such as image recognition and classification. CNN architecture is different compared when compared with regular neural networks. A typical CNN has three layers namely such as convolution, pooling, and fully connected layers. The core building block is the convolution layer which transfers the network computation load. By applying a large number of kernels the input image is convoluted. By pooling the values in adjacent pixels each feature map is downsized to a smaller matrix. [12].

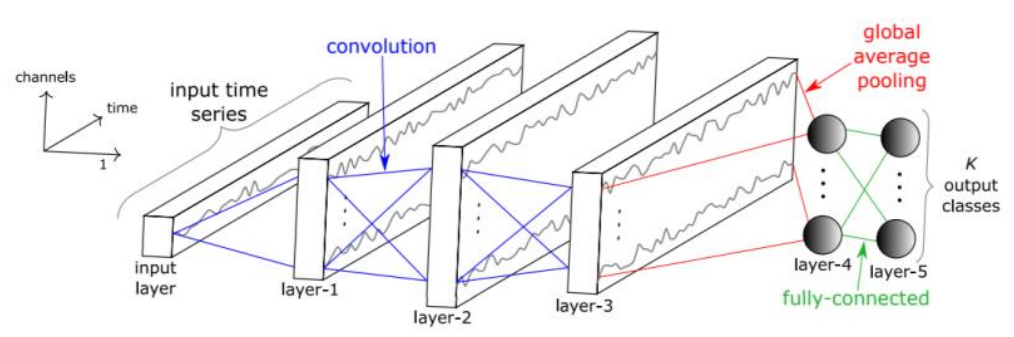

Fig.3.Fully Convolutional Neural Network Architecture

\subsection{AlexNet}

The AlexNet architecture $[13,14,15]$ is shown in Fig 4. AlexNet is a convolutional neural network consist of eight layers. The initial five layers are convolutional and the rest three are fully connected layers. ReLU is cast-off after every convolutional and fully connected layer. To add non-linearity Rectified Linear Units is applied despite the Tanh function. The speed is increased by 6 times with the same accuracy. Dropout is equipped before the first two fully connected layers. To avoid overfitting dropout is equipped despite regularisation. Hence the dropout rate of 0.5 is doubled in training time. The network size is reduced by adding overlap pooling. AlexNet is used in various applications not limited to medical image processing, natural language processing and other image processing tasks. 


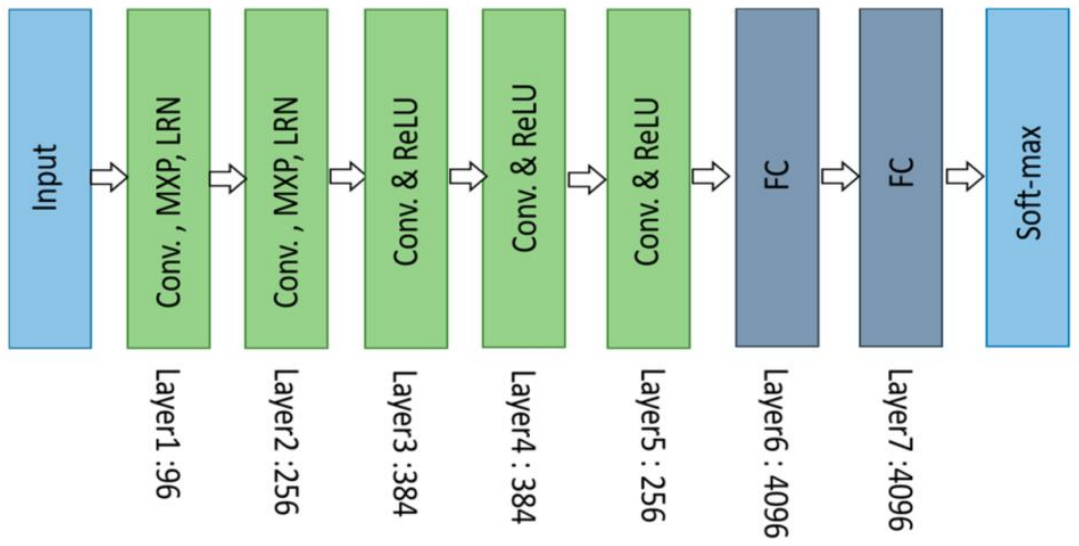

Fig. 4. Architecture of AlexNet. [14]

Table 1.Prediction Evaluation Metrics by AlexNet

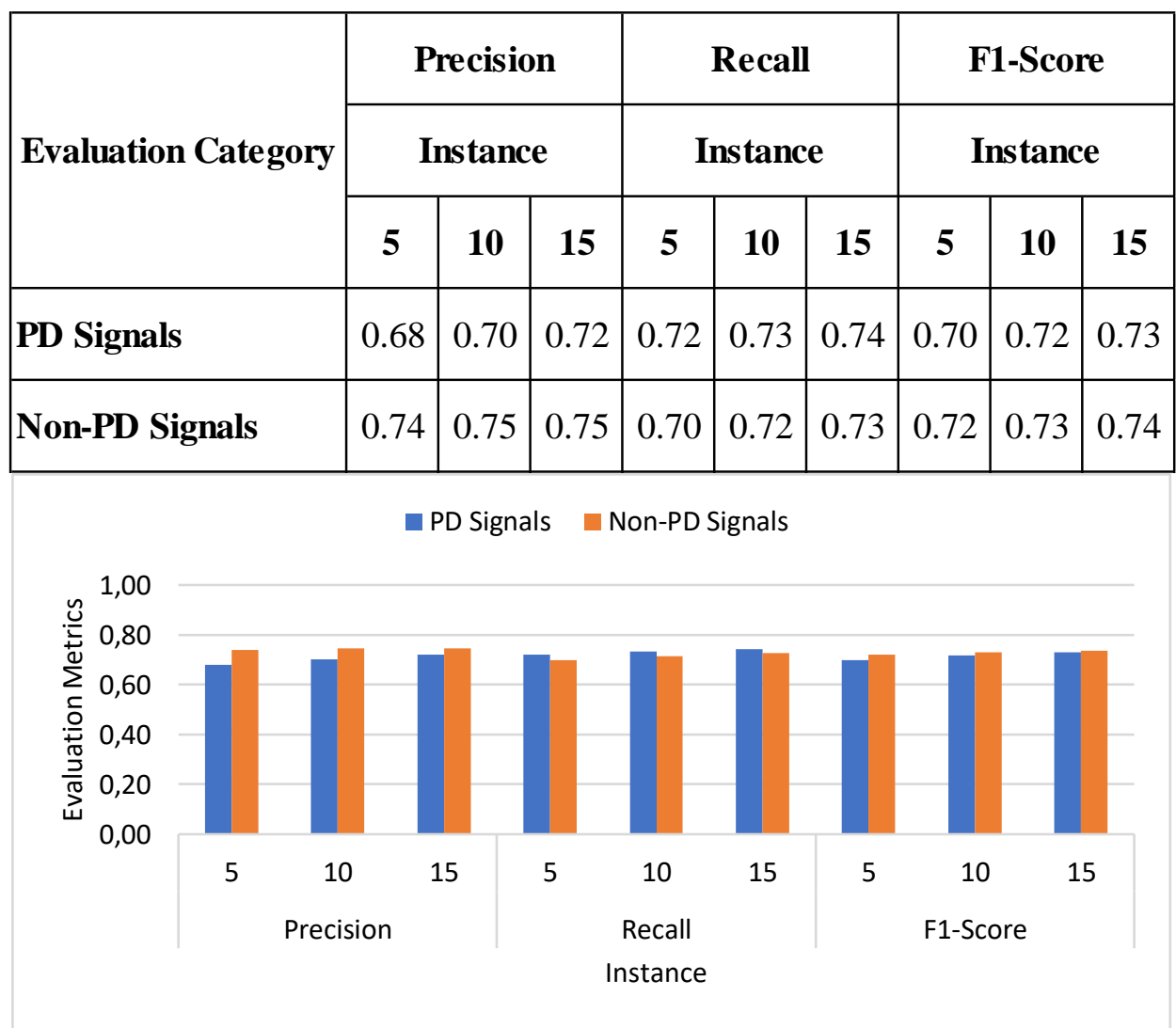

Fig.5.Prediction Evaluation Metrics Plot for AlexNet

Based on the performance evaluation metrics of AlexNet architecture is shown in Table 1 $\&$ Figure 5. At the various instances of 5, $10 \& 15$, it is observed that Precision \& Recall values shows $68 \%, 70 \% \& 72 \%$ of detected PD signals which are true PD signals out of $72 \%$, $73 \%$ \& $74 \%$ successfully detected PD signals the F1-Score at the various instances are $70 \%$, $72 \%$ \& $73 \%$ respectively. 


\subsection{VGG-16}

The VGG-16 Network is shown in Fig 5. It consists of 16 layers with some weights. The input is an image with a dimension of $224 \times 224 \times 3$. The initial 2 layers have $2 \times$ Convolution \& Max Polling layer with 64 channel feature map size of 244 x 224 x 64 and 112 x 112 x x 64 dimensions. The $3^{\text {rd }} \& 4^{\text {th }}$ layers have 2 x Convolution $\&$ Max Polling layer with 128 channel feature map size of $112 \times 112 \times 128$ and $56 \times 56 \times 128$ dimensions. The $5^{\text {th }} \& 6^{\text {th }}$ layers have 2 x Convolution \& Max Polling layer with 256 channel feature map size of 56 x 56 x 256 and 28 x $28 \times 256$ dimensions. The $7^{\text {th }} \& 8^{\text {th }}$ layers have $3 \times$ Convolution $\&$ Max Polling layer with 512 channel feature map size of $28 \times 28 \times 512$ and 14 × 14 × 512 dimensions. The last convolution layers have 3 x Convolution \& Max Polling layer with 512 channel feature map size of $14 \times 14 \times 512 \& 7 \times 7 \times 512$ dimensions. All the layers have $3 \times 3$ Kernel size with a stride of $(1,2)$ respectively. Followed by three Fully connected layers with a size of 25088 , 4096 \& 4096 are used. ReLU activation function is cast off in all hidden layers. The vanishing gradient problem is also decreased by using ReLU it also increases the learning time which leads to efficient computing. The output layer is also a fully connected layer with a size of 1000 and SoftMax as an activation function.

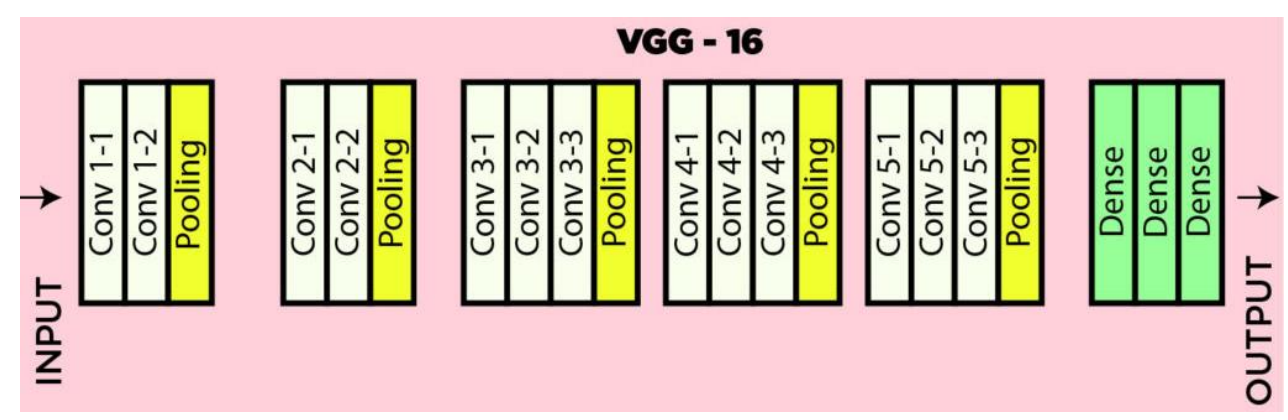

Fig.6.VGG-16 Network Architecture. [15,16]

Based on the performance evaluation metrics of VGG-16 architecture is shown in Table 2 $\&$ Figure 7. At the various instances of $5,10 \& 15$, it is observed that Precision \& Recall values shows $74 \%, 75 \% \& 78 \%$ of detected PD signals which are true PD signals out of $76 \%$, $78 \%$ \& $79 \%$ successfully detected PD signals the F1-Score at the various instances are $75 \%$, $76 \%$ \& $78 \%$ respectively.

Table 2 Prediction Evaluation Metrics by VGG-16

\begin{tabular}{|l|c|c|c|c|c|c|c|c|c|}
\hline \multirow{2}{*}{ Evaluation Category } & \multicolumn{3}{|c|}{ Precision } & \multicolumn{3}{c|}{ Recall } & \multicolumn{3}{c|}{ F1-Score } \\
\cline { 2 - 10 } & \multicolumn{2}{|c|}{ Instance } & \multicolumn{2}{c|}{ Instance } & \multicolumn{3}{c|}{ Instance } \\
\cline { 2 - 10 } & $\mathbf{5}$ & $\mathbf{1 0}$ & $\mathbf{1 5}$ & $\mathbf{5}$ & $\mathbf{1 0}$ & $\mathbf{1 5}$ & $\mathbf{5}$ & $\mathbf{1 0}$ & $\mathbf{1 5}$ \\
\hline PD Signals & 0.74 & 0.75 & 0.78 & 0.76 & 0.78 & 0.79 & 0.75 & 0.76 & 0.78 \\
\hline Non-PD Signals & 0.77 & 0.78 & 0.78 & 0.74 & 0.76 & 0.77 & 0.76 & 0.77 & 0.78 \\
\hline
\end{tabular}




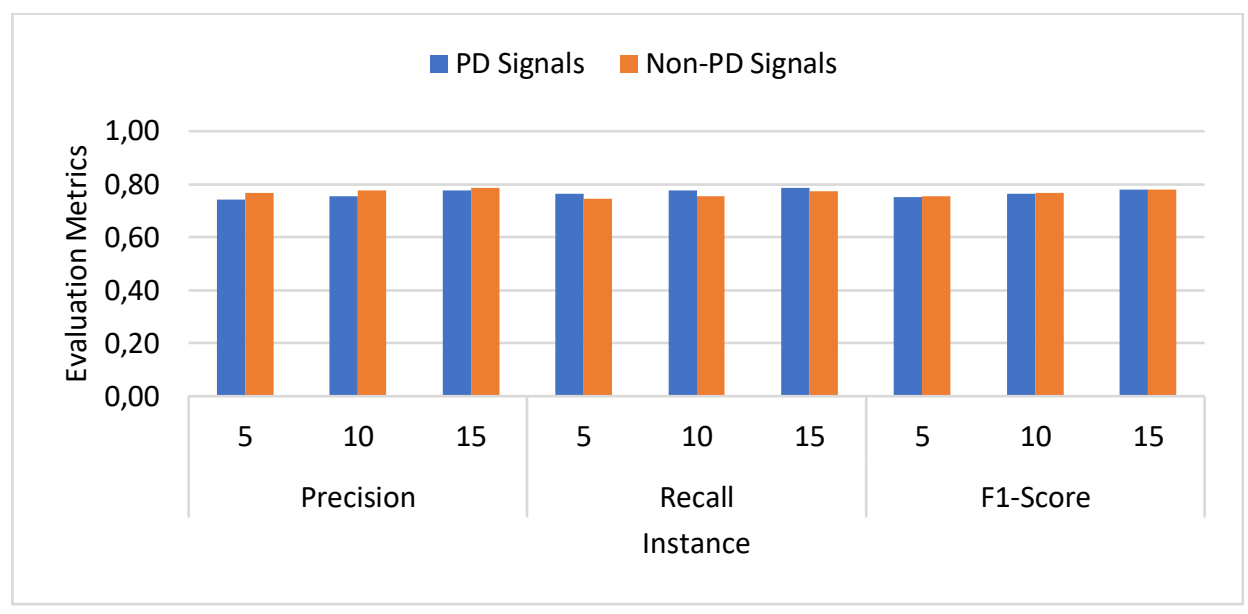

Fig. 7.Prediction Evaluation Metrics Plot for VGG-16

\subsection{Residual Network (ResNet)}

ResNet $[17,18,19]$ shown in Fig 4. is a type of deep neural networks with 150+ layers. It is frequently used in many computer vision tasks. Before ResNet due to the problem of vanishing gradients training of deep neural network is problematic. To overcome the vanishing gradient problem, skip connection is used in ResNet. Layers such as 34, 50,101, 152, and even 1202 are used in ResNet. It consists of 49 convolution and 1 fully connected layer. ResNet is built with the total number of $25.5 \mathrm{M}$ weights \& $3.9 \mathrm{M}$ Multiply and Accumulates (MACs).

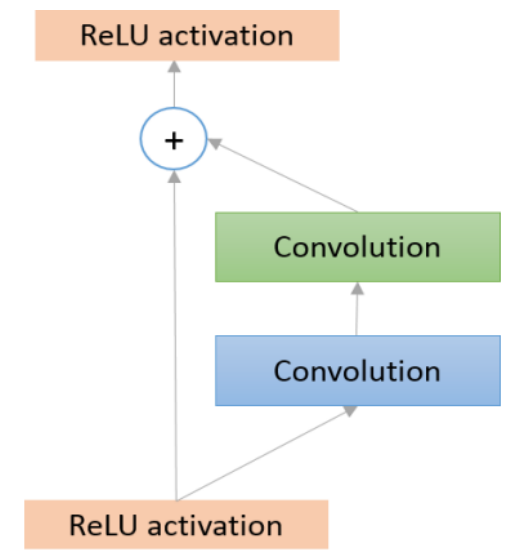

Fig. 8.Residual block. [19]

Based on the performance evaluation metrics of ResNet architecture is shown in Table 3 $\&$ Figure 9. At the various instances of $5,10 \& 15$, it is observed that Precision \& Recall values shows $79 \%, 80 \%$ \& $80 \%$ of detected PD signals which are true PD signals out of $80 \%$, $82 \% \& 81 \%$ successfully detected PD signals the F1-Score at the various instances are $79 \%$, $79 \%$ \& $81 \%$ respectively. 
Table 3 Prediction Evaluation Metrics by ResNet

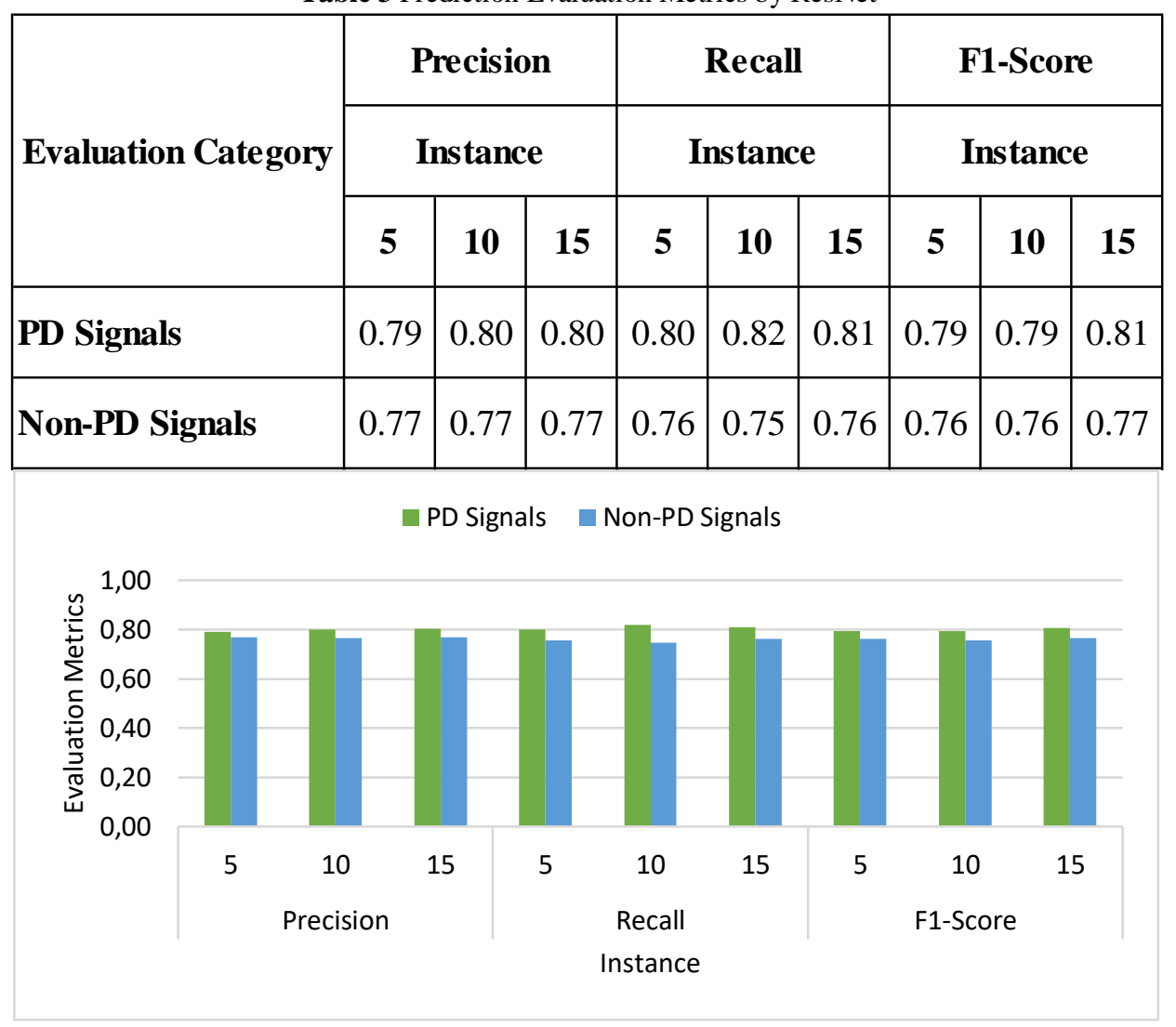

Fig.9.Prediction Evaluation Metrics Plot for ResNet

\section{Result \& Discussion:}

At the various instance of $5,10 \& 15$, it is observed that Precision \& Recall values shows $70 \%, 72 \% \& 73 \%$ of detected PD signals which are true PD signals out of $75 \%, 76 \% \& 78 \%$ successfully detected PD signals the F1-Score at the various instances are 79\%, 79\% \& $81 \%$ respectively. Based on the analysis of Prediction Evaluation Metrics shown in Table $4 \&$ Figure 10 for different pre-trained CNN Architectures such as AlexNet, VGG-16 \& ResNet, it is observed that ResNet architecture provides the best prediction and classification results.

Table 4 F1-Score of different pre-trained CNN Architectures

\begin{tabular}{|l|c|c|c|c|c|c|c|c|c|}
\hline \multirow{3}{*}{ F1-Score } & \multicolumn{2}{|c|}{ AlexNet } & \multicolumn{3}{c|}{ VGG-16 } & \multicolumn{3}{c|}{ ResNet } \\
\cline { 2 - 10 } & \multicolumn{2}{|c|}{ Instance } & \multicolumn{3}{c|}{ Instance } & \multicolumn{3}{c|}{ Instance } \\
\cline { 2 - 10 } & $\mathbf{5}$ & $\mathbf{1 0}$ & $\mathbf{1 5}$ & $\mathbf{5}$ & $\mathbf{1 0}$ & $\mathbf{1 5}$ & $\mathbf{5}$ & $\mathbf{1 0}$ & $\mathbf{1 5}$ \\
\hline PD Signals & 0.70 & 0.72 & 0.73 & 0.75 & 0.76 & 0.78 & 0.79 & 0.79 & 0.81 \\
\hline Non-PD Signals & 0.72 & 0.73 & 0.74 & 0.76 & 0.77 & 0.78 & 0.76 & 0.76 & 0.77 \\
\hline
\end{tabular}




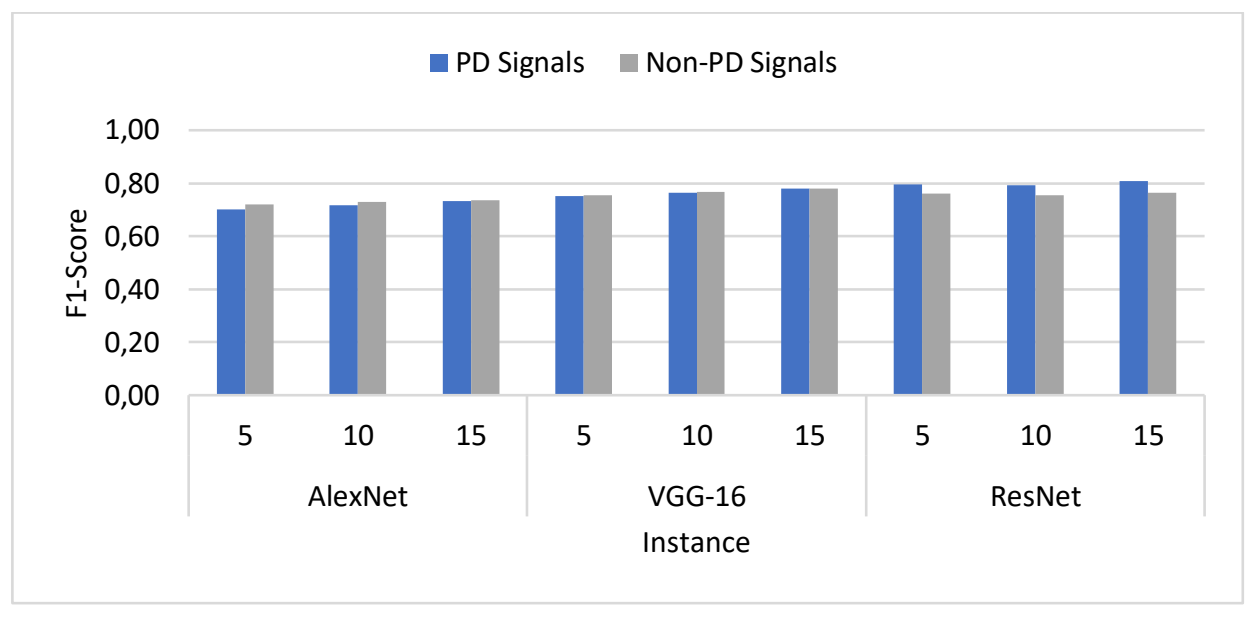

Fig.10.F1-Score Plot for different pre-trained CNN Architectures

\section{Conclusion}

In this paper, the contemporary development made on the application of CNN techniques for identifying PD sources are discussed. CNN based models for the classification of time series has numerous significant benefits over other methods. To analyze and classify the raw voltage signal for the detection of PD's in IOC's a CNN based fault classification algorithm is proposed. The CNN is implemented using popular pre-trained CNN architectures such as AlexNet, VGG16 \& ResNet are applied to the voltage signals in the dataset. The trained classifier will be able to detect future PDs in IOC's. From the values of Precision, Recall \& F1-Score it is observed that ResNet architecture provides the best prediction and classification results.

\section{References:}

1. W. ReklamníagenturaMARF, "ENET Centre - Introduction | ENET Centre | Centrum ENET", Cenet.vsb.cz, 2020. [Online]. Available: http://cenet.vsb.cz/en/informace/enet-centre/160/enetcentre-introduction.html. [Accessed: 21- Jun- 2020].

2. Baskar. D, Selvam. P, "Electrical Transmission Line Fault Detection and Classification using Convolution Neural Networks and Support Vector Machine," Test Engineering \& Management, vol. 82, pp. 6803-6808, Jan./Feb. 2020.

3. N. Qu, Z. Li, J. Zuo and J. Chen, "Fault Detection on Insulated Overhead Conductors Based on DWT-LSTM and Partial Discharge," IEEE Access, vol. 8, pp. 87060-87070, 2020.

4. M. Dong and J. Sun, "Partial discharge detection on aerial covered conductors using time-series decomposition and long short-term memory network," Electric Power Systems Research, vol. 184, p. 106318, Mar. 2020.

5. Baskar, D. " Efficient sequential switching hybrid modulation techniques for cascaded multilevel inverters Middle - East Journal of Scientific Research, 2014, 20(12), pp. 2523-2528

6. "VSB Power Line Fault Detection | Kaggle", Kaggle.com, 2020. [Online]. Available: https://www.kaggle.com/c/vsb-power-line-fault-detection/data. [Accessed: 21- Jun- 2020].

7. Baskar. D, Selvam. P, "Machine Learning framework for Power System Fault Detection and Classification," International Journal of Scientific \& Technology Research, vol. 9, no. 2, pp. 2002 2008, Feb. 2020.

8. "Apache Parquet", Parquet.apache.org, 2020. [Online]. Available: https://parquet.apache.org/documentation/latest/. [Accessed: 21- Jun- 2020].

9. Charles Rajesh Kumar, J., Vinod Kumar, D., Baskar, D., ...Jenova, R., Majid, M.A. "Offshore wind energy status, challenges, opportunities, environmental impacts, occupational health, and safety management in India" Energy and Environment, 2020

10. Baskar, D. " Current control of boost single phase PFC converters" Middle - East Journal of Scientific Research, 2014, 19(9), pp. 1250-1258.

11. R.S. Michalski, J.G. Carbonell, T.M. Mitchell, Machine Learning: An Artificial Intelligence Approach, Springer Science \& Business Media, 2013.

12. B. Zhao, H. Lu, S. Chen, J. Liu and D. Wu, "Convolutional neural networks for time series classification," Journal of Systems Engineering and Electronics, vol. 28, no. 1, pp. 162-169, Feb. 2017. 
13. Muthukrishnan, A.; Charles Rajesh Kumar, J.; Vinod Kumar, D.; Kanagaraj, M. Internet of image things-discrete wavelet transform and Gabor wavelet transform based image enhancement resolution technique for IoT satellite applications. Cogn. Syst. Res. 2019, 57, 46-53.

14. M. Z. Alom, T. M. Taha, C. Yakopcic, S. Westberg, P. Sidike, M. S. Nasrin, M. Hasan, B. C. Van Essen, A. A. S. Awwal, and V. K. Asari, "A State-of-the-Art Survey on Deep Learning Theory and Architectures," Electronics, vol. 8, no. 3, p. 292, Mar. 2019.

15. Charles Rajesh Kumar.J , Vinod Kumar.D, Baskar.D, Mary Arunsi.B, Jenova.R, ,M.A.Majid." VLSI design and implementation of High-performance Binary-weighted convolutional artificial neural networks for embedded vision based Internet of Things (IoT)".16th International Learning \& Technology Conference (L\&T), Procedia Computer science (Elsevier), Jeddah,2018.

16. K. Simonyan and A. Zisserman, "Very deep convolutional networks for large-scale image recognition," arXiv preprint arXiv:1409.1556, 2014.

17. Md ZahangirAlom , Tarek M. Taha , Chris Yakopcic, Stefan Westberg, PahedingSidike , MstShamima Nasrin , Brian C Van Essen , Abdul A S. Awwal , and Vijayan K. Asari, "The History Began from AlexNet: A Comprehensive Survey on Deep Learning Approach" arXiv:1803.01164, 2018.

18. Charles Rajesh Kumar, J., Vinod Kumar, D., Majid, M.A. "Wind energy programme in India: Emerging energy alternatives for sustainable growth" Energy and Environment, 2019, 30(7), pp. $1135-1189$

19. K. He, X. Zhang, S. Ren, and J. Sun, "Deep Residual Learning for Image Recognition,” 2016 IEEE Conference on Computer Vision and Pattern Recognition (CVPR), Las Vegas, NV, USA, June 2016; pp. 770-778.

20. T. Vijayan , M. Sangeetha , A. Kumaravel \& B. Karthik (2020): FeatureSelection for Simple Color Histogram Filter based on Retinal Fundus Images for DiabeticRetinopathy Recognition, IETE Journal of Research, DOI: 10.1080/03772063.2020.1844082.

21. D. S. Vijayan, A. Leema Rose, S. Arvindan, J. Revathy, C. Amuthadevi, "Automation systems in smart buildings: a review", Journal of Ambient Intelligence and Humanized Computing https://doi.org/10.1007/s12652-020-02666-9

22. Vijayan T, Sangeetha M, A. Kumaravel, Karthik B, "Gabor filter and machine learning based diabetic retinopathy analysis and detection”, Microprocessors and Microsystems,2020. https://doi.org/10.1016/j.micpro.2020.103353.

23. Vijayan T, SangeethaM, Karthik B, "Trainable WEKA Segmentation of Retinal Fundus Images for Global Eye Disease Diagnosis Application," International Journal of Emerging Trends in $\begin{array}{llllll}\text { Engineering Research,Vol } & \text { 8, No.9, pp. 5750-5754, } & \text { Sep } 2020 .\end{array}$ https://doi.org/10.30534/ijeter/2020/136892020

24. C. Amuthadevi, D. S. Vijayan, Varatharajan Ramachandran, "Development of air quality monitoring (AQM) models using different machine learning approaches", Journal of Ambient Intelligence and Humanized Computing, https://doi.org/10.1007/s12652-020-02724-2

25. Vijayan T, Sangeetha M, A. Kumaravel, Karthik B, "Fine Tuned VGG19 Convolutional Neural Network Architecture for Diabetic Retinopathy Diagnosis," Indian Journal of Computer Science and Engineering (IJCSE), Vol. 11, No. 5, pp. 615-622 Sep-Oct 2020. DOI: 10.21817/indjcse/2020/v11i5/201105266.

26. Vijayan T, Sangeetha M, Karthik B, "Efficient Analysis of Diabetic Retinopathy on Retinal Fundus Images using Deep Learning Techniques with Inception V3 Architecture,” Journal of Green Engineering, Vol 10, Issue 10, pp. 9615-9625. Oct 2020 